\title{
Experimental and Theoretical IR Spectra of 2-Nitrosopyridines
}

\author{
Katarina Varga, ${ }^{1}$ (1) Hrvoj Vančik, ${ }^{2}$ 이 Zlatko Mihalić2,*
}

\footnotetext{
1 Institute of Organic Chemistry and Biochemistry of the Czech Academy of Sciences, Flemingovo náměstí 542/2, 16000 Praha 6, Czech Republic

2 Department of Chemistry, Faculty of Science, University of Zagreb, Horvatovac 102a, 10000 Zagreb, Croatia

* Corresponding author's e-mail address: mihalic@chem.pmf.hr
}

RECEIVED: July 16, 2021 * REVISED: August 4, $2021 \star$ ACCEPTED: August 4, 2021

— This Paper is Dedicated to Prof. Milan Randić on the occasion of his 90 ${ }^{\mathrm{Th}}$ Birthday, and to the Memory of Prof. Mircea Diudea

Abstract: Previously unknown IR spectra of 2-nitrosopyridines 1-3 were recorded in the solid phase after cryogenic photolysis of their dimers, and identified by the comparison with the corresponding theoretical spectra. Optimal results were obtained with double-hybrid (DH) density functionals and def2-TZVP basis set, the best being DSD-PBEP86-D3(BJ), B2GP-PLYP-D3(BJ) and mPW2-PLYP-D2. Experimental frequencies were obtained by scaling harmonic frequencies with appropriate scaling factors, either taken from the literature or calculated using modified HFREQ2014 dataset. In the case of DH functionals, anharmonic frequencies do not offer any advantage in comparison with the scaled harmonic ones. Unexpectedly, all non-DH density functionals, including highly praised $\omega B$ B7X-V and $\omega B 97 M-V$, have serious problems with normal modes containing $\mathrm{N}=\mathrm{O}$ bond stretching vibration. It was concluded that the origin of the problem is in the nitroso group itself, most likely caused by its multireference character. The best results among non-DH functionals were achieved at TPSSh-D3(BJ)/def2-TZVP level of theory.

Keywords: cryogenic photolysis, 2-nitrosopyridine, nitroso monomers, vibrational spectra, double-hybrid density functionals, DSD-PBEP86$\mathrm{D} 3(\mathrm{BJ})$, harmonic frequencies, anharmonic frequencies, scaling factors.

\section{INTRODUCTION}

C NITROSO compounds exist in two forms, as monomers and as dimers-azodioxides. ${ }^{[1]}$ Since in the solid state most of them appear as dimers, it is difficult to record and interpret the IR spectra of monomeric forms. This became much easier after the development of method by which the monomers in solid state were prepared by photolysis of azodioxide polycrystals in $\mathrm{KBr}$ pellet at cryogenic temperatures (12-23 K). ${ }^{[2,3]}$ Although nitroso monomers have so far routinely been identified by strong IR absorbance in the spectral region near to $1500 \mathrm{~cm}^{-1}$, assigned to $\mathrm{N}=\mathrm{O}$ stretching vibration, ${ }^{[1]}$ the detailed interpretation of spectra of these compounds has never been reported.

In this work we report FTIR spectra of three 2-nitrosopyridines (1-3, Scheme 1 ) recorded after the photolysis of their dimers in $\mathrm{KBr}$ pellet at $12 \mathrm{~K}$, as well as their identification and partial assignations by the comparison of observed frequencies with the vibrational frequencies calculated by the density functional theory (DFT) approach.

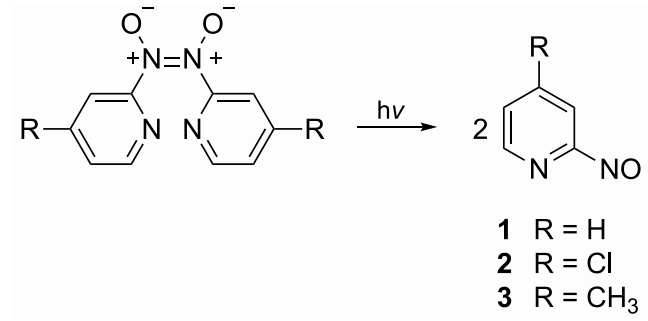

Scheme 1.

Also, as the DFT results proved to be quite density functional dependent, molecules containing nitroso group emerged as challenging model for testing various functionals' ability to produce accurate theoretical vibrational frequencies. A number of density functionals and basis sets were employed for the calculation of harmonic and anharmonic vibrational frequencies of three 2-nitrosopyridines (1-3) and nitrosobenzene, ${ }^{[3]}$ and the results compared between themselves and with the experimental frequencies to find the optimal level of theory for the job in hand.

(cc) Br 


\section{EXPERIMENTAL}

\section{Preparation of Compounds}

Dimers of 2-nitrosopyridines 1-3 were prepared as reported before. ${ }^{[4]}$

\section{Spectroscopic Methods}

For FTIR measurements Bruker Equinox FTIR spectrometer with $1 \mathrm{~cm}^{-1}$ resolution was used. The sample was prepared as standard $\mathrm{KBr}$ pellet cooled by Leybold-Heraeus ROK 10-300 cyclic helium cryostat. Photolysis of crystal were conducted by a high-pressure Hg lamp (250 W).

\section{Computational Methods}

For geometrical optimizations and calculations of vibrational frequencies three quantum chemical programs were used: Gaussian 16 rev. C.01, ${ }^{[5]}$ Orca 4.2.1 ${ }^{[6]}$ and Q-Chem 5.1.2. ${ }^{[7]}$ Most of the calculations were done with Gaussian 16 because it has implemented analytical second derivatives for all its functionals, which significantly speeds up frequency jobs. In four cases harmonic frequencies had to be calculated numerically, SCS-MP2 and B2GP-PLYP-D3(BJ) frequencies with Orca, and $\omega B 97 X-V$ and $\omega B 97 M-V$ frequencies with Q-Chem.

Geometry optimizations with all three programs were conducted using tighter optimization criteria ('opt=tight' in Gaussian, 'tightopt' in Orca, and equivalent in Q-Chem), and denser integration grids ('int=ultrafine' or 'int=superfine' in Gaussian, 'Grid6' in Orca and 'SG-3' in QChem).

Harmonic vibrational normal modes obtained with Gaussian and Orca were analyzed with program vibAnalysis. ${ }^{\left[{ }^{8]}\right.}$ Based on VMARD method, ${ }^{[9]}$ it uses Bayesian linear regression to decompose vibrational normal modes into a small number of the most prominent internal coordinates describing individual atomic motions. Comparison and analysis of harmonic and anharmonic spectra obtained with Gaussian program was also done with GaussView v6. ${ }^{[10]}$

Theoretical infrared spectra were calculated from scaled harmonic or anharmonic frequencies by in-house program using Lorentzian lineshape function with peak half-width at half height $4 \mathrm{~cm}^{-1}$, and plotted with graphing program DataGraph. ${ }^{[11]}$

Scaling factors for obtaining fundamental frequencies from harmonic frequencies were calculated following the procedure described by Martin, ${ }^{[12]}$ using frequency dataset from the same paper (modified HFREQ2014) containing 30 molecules with total of 119 experimental fundamental frequencies. Fitting was done with program DataGraph.

\section{RESULTS AND DISCUSSION}

\section{Solid-State Photolysis of 2-Nitrosopyridines Dimers 2-NITROSOPYRIDINE (1)}

FTIR spectrum of 2-nitrosopyridine dimer recorded in $\mathrm{KBr}$ pellet at $12 \mathrm{~K}$ exhibits intense signals at $1390 \mathrm{~cm}^{-1}$ and $1409 \mathrm{~cm}^{-1}$ assigned to the symmetric and asymmetric stretching vibrations of the Z-ONNO group ${ }^{[13]}$ (Figure 1a). After photolysis by a high-pressure $\mathrm{Hg}$ lamp, the intensities of these signals decrease and several new signals appear (Figure 1b). The most prominent of them at $1509 \mathrm{~cm}^{-1}$ can be tentatively assigned to the stretching vibration of $\mathrm{N}=\mathrm{O}$ group, indicating formation of monomer 1 . By warming the sample to the room temperature, the signals assigned to dimer restore their intensity, and the signals of the monomer disappear (Figure 1c).

\section{4-CHLORO-2-NITROSOPYRIDINE (2)}

Similarly as with $\mathbf{1}$, irradiation of dimer of $\mathbf{2}$ by a high-pressure $\mathrm{Hg}$ lamp in $\mathrm{KBr}$ pellet at $12 \mathrm{~K}$ induces its photodissociation to monomers. FTIR spectra show that starting mixture of $Z$ - and $E$ - dimers also undergoes photodissociation in $\mathrm{KBr}$ pellet at $12 \mathrm{~K}$. Intensities of the signals of $Z$ - and E-ONNO groups at 1389 and $1250 \mathrm{~cm}^{-1}$ decrease upon irradiation, whereas the new signal attributed to monomeric $\mathrm{N}=\mathrm{O}$ group appears at $1507 \mathrm{~cm}^{-1}$. Both $Z$ - and $E$ dimers are regenerated by thermal dimerization.

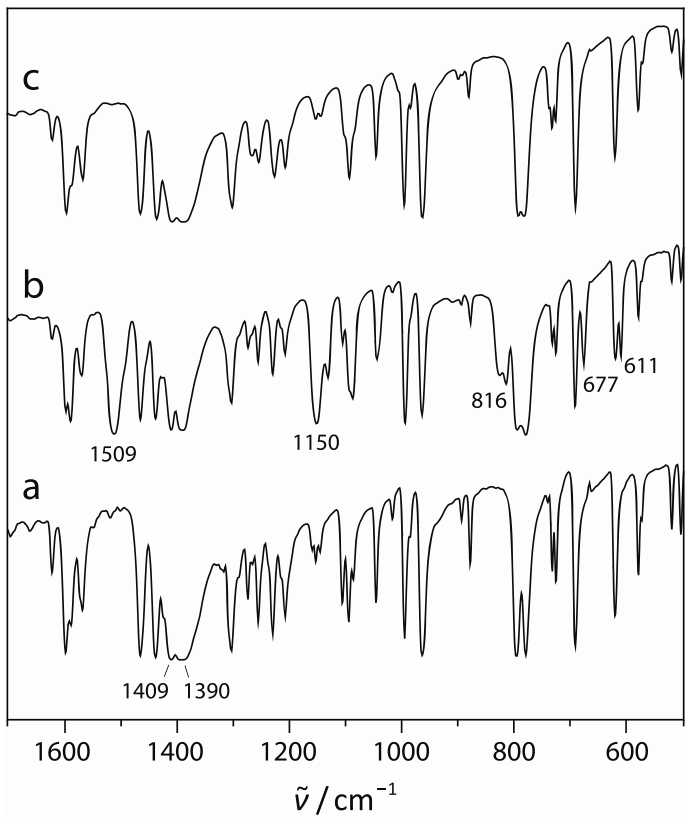

Figure 1. FTIR spectra of 1 in $\mathrm{KBr}$ pellet (a) at $12 \mathrm{~K}$, (b) at $12 \mathrm{~K}$ after photolysis by a high-pressure $\mathrm{Hg}$ lamp, and (c) after warming to room temperature. 


\section{4-METHYL-2-NITROSOPYRIDINE (3)}

Dimer of compound $\mathbf{3}$ affords solid-state photo behavior similar to 2-nitrosopyridine and its 4-chloro derivative 2. Intensities of the signals of $Z$ - and $E$-dimers at 1400 and $1266 \mathrm{~cm}^{-1}$ are reduced upon irradiation and a new intensive band that can be assigned to stretching vibration of monomeric $\mathrm{N}=\mathrm{O}$ group appears at $1512 \mathrm{~cm}^{-1}$. Warming the sample to the room temperature regenerates the starting spectrum. Appearance of monomers in solid phase after cryogenic photolysis opens opportunity to record and examine their yet unknown vibrational spectra. In Figure 2 are given differential FTIR spectra obtained after photolysis of corresponding dimers. All three of them contain a strong band between 1509 and $1512 \mathrm{~cm}^{-1}$, and since it is known that nitroso compounds have $\mathrm{N}=\mathrm{O}$ bond stretching band in this region, ${ }^{[1]}$ it is reasonable to assume that irradiation of dimers produces corresponding monomers. The best way to confirm it is to compare theoretical vibrational frequencies of monomers with the frequencies of the bands in experimentally recorded differential spectra.

\section{Calculation of Vibrational Spectra of Monomers}

There are two standard ways to calculate vibrational frequencies of molecules. Simpler and more often used is based on harmonic force field approximation, in which

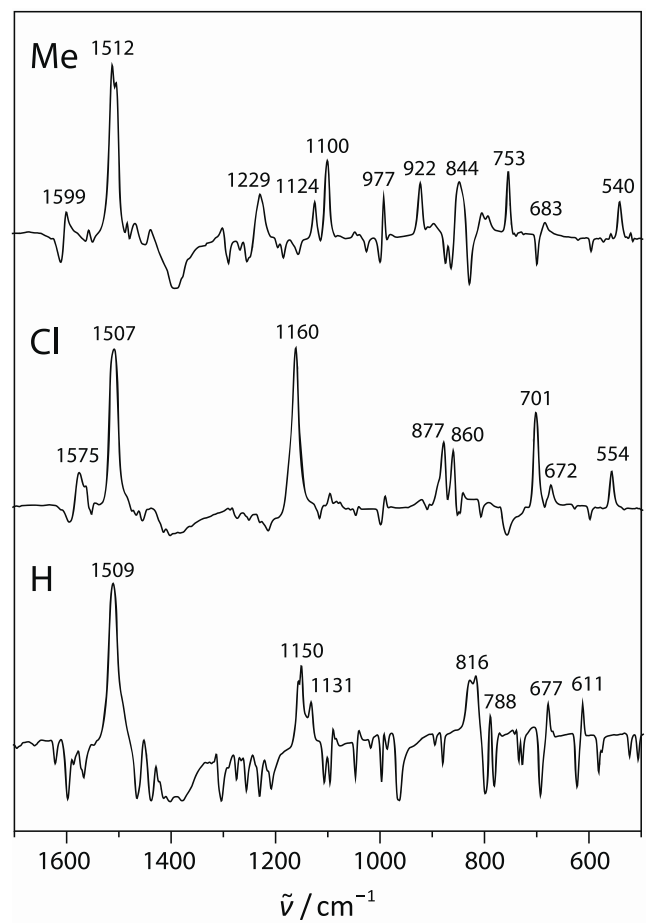

Figure 2. Differential spectra of 2-nitrosopyridines 1-3 recorded after photolysis in $\mathrm{KBr}$ pellet at $12 \mathrm{~K}$. Newly formed bands are pointing upwards. Each shown wavenumber is an average of several independent measurements. potential energy in the vicinity of stationary point is expanded into Taylor series and truncated after second order term. This allows calculation of harmonic vibrational frequencies by diagonalization of the massweighted second order energy derivatives matrix. Due to anharmonicity effects, electron correlation effects, and basis sets incompleteness, frequencies calculated in this way are higher than the experimental fundamental frequencies. The simplest of several ways ${ }^{[14]}$ to deal with this problem is to multiply calculated harmonic frequencies by a single scaling factor specific for the particular level of theory.

More accurate approach, though rather lengthy one, is to calculate anharmonic corrections to the vibrational energy using second-order vibrational perturbation theory (VPT2). ${ }^{[15]}$ As it requires third and fourth order energy derivatives with respect to the normal coordinates, the procedure is feasible only if used quantum mechanical model has implemented analytical second derivatives.

We have chosen to calculate vibrational frequencies of monomers using density functional theory. Since preliminary calculations revealed that calculated 2-nitrosopyridines frequencies are quite density functional dependent, a larger number of functionals were tested to find the ones that produce reliable results. Functionals were selected for testing either because of their popularity ${ }^{[16]}$ and widespread presence in the literature, or because they were well positioned in reliable benchmark studies of density functionals performance. ${ }^{[17]}$ In the interest of efficiency, for the calculation of harmonic vibrational frequencies we have considered only functionals for which at least analytical first derivatives were available, and for the calculation of anharmonic frequencies functionals with analytical second derivatives as well.

In combination with the selected functionals, several families of basis sets were tested: Pople's $s^{[18]} 6$ $311+G(d, p)$ and $6-311+G(2 d f, 2 p)$, Dunning's $c c-p V n Z$ and aug-cc-pVnZ,, ${ }^{[19]}$ Jensen's PCSEG- $n$ and aug-PCSEG- $n,{ }^{[20]}$ and Karlsruhe def2 ${ }^{[21]}$ basis sets of double, triple and quadruple- $\zeta$ quality. Comparison of spectra of nitroso compounds calculated with different members of the same basis sets family demonstrated that spectra basically do not change beyond triple- $\zeta$ level, and do not change with the addition of diffuse functions. Since MP2 calculations were performed as well, the advantage was given to def2-TZVP basis set, whose overall performance was the most consistent of all tested basis sets.

So far, scaling factors for conversion of calculated harmonic to fundamental frequencies for def2 basis sets have been published, to the best of our knowledge, only by Truhlar ${ }^{[22]}$ and Martin. ${ }^{[12]}$ Truhlar's scaling factors for various density functionals and mainly def2-TZVP basis set were based on F38/10 dataset consisting of 38 experimental fundamental frequencies of 15 molecules. Martin's 
scaling factors for MP2, SCS-MP2, and a variety of density functionals were based on modified HFREQ2014 dataset consisting of 119 experimental fundamental frequencies of 30 molecules. The rest of scaling factors used in this paper were determined by ourselves using Martin's procedure and his dataset given in ref. 12 .

\section{Comparison of Calculated and Experimental Frequencies}

The main difference between different functionals are the frequencies and intensities of the normal modes containing $\mathrm{N}=\mathrm{O}$ stretching vibration. While spectra calculated with the most of double-hybrid functionals look just like the experimental spectra, spectra calculated with other functionals significantly deviate, suggesting that not all functionals are capable of predicting accurate vibrational frequencies of these compounds.

Another potential complication is that 2-nitrosopyridines have two conformers, syn and anti (Scheme 2), making the analysis of their experimental spectra more difficult.

On all tested DFT levels of theory anti conformers of 2-nitrosopyridines 1-3 are for $0.4-0.7 \mathrm{kcal} \mathrm{mol}^{-1}$ more stable than corresponding syn conformers. In Table 1 are given their relative standard Gibbs energies, and barriers for gas-phase interconversion by out-of-plane rotation of $\mathrm{N}=\mathrm{O}$ group around $\mathrm{C}-\mathrm{N}(=\mathrm{O})$ bond, all calculated at DSDPBEP86-D3(BJ)/def2TZVP level of theory.

Since in solid state pyridine nitrogens in Z-dimer are syn, and in E-dimer anti to each other, ${ }^{[4]}$ one would expect that cryogenic photolysis in both cases produces at least predominantly anti-2nitrosopyridine (Scheme 3). Especially since at $12 \mathrm{~K}$ isomerization is very slow, and the difference

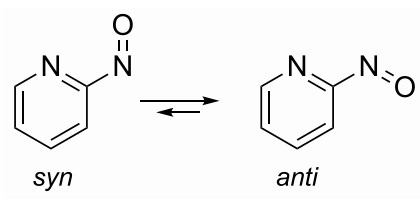

Scheme 2.

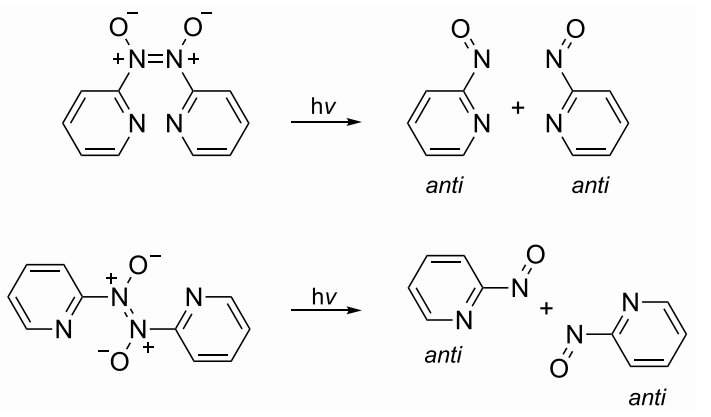

Scheme 3.
Table 1. Relative standard Gibbs energies (in $\mathrm{kcal} \mathrm{mol}^{-1}$ ) at $298.15 \mathrm{~K}$ and $12 \mathrm{~K}$ of anti and syn conformers of three 2-nitrosopyridines (1-3) and nitrosobenzene, and transition states (TS) for their gas-phase interconversion, calculated at DSD-PBEP86-D3(BJ)/def2-TZVP level of theory.

\begin{tabular}{ccc}
\hline & $G^{\circ}(298.15 \mathrm{~K})_{\text {rel }}$ & $G^{\circ}(12 \mathrm{~K})_{\text {rel }}$ \\
\hline 2-nitrosopyridine $(\mathrm{R}=\mathrm{H})$ & & \\
anti & 0.00 & 0.00 \\
syn & 0.61 & 0.66 \\
TS & 5.69 & 5.13 \\
4-methyl-2-nitrosopyridine $(\mathrm{R}=\mathrm{Cl})$ & \\
anti & 0.00 & 0.00 \\
syn & 0.66 & 0.72 \\
TS & 5.55 & 4.99 \\
4-methyl-2-nitrosopyridine $(\mathrm{R}=\mathrm{CH})$ & \\
Anti & 0.00 & 0.00 \\
Syn & 0.39 & 0.62 \\
TS & 5.85 & 5.14 \\
nitrosobenzene & & \\
min & 0.00 & \\
TS & 7.83 &
\end{tabular}

in stabilities is sufficient that equilibrium mixture contains virtually no syn conformer.

Scaled harmonic infrared spectra of anti and syn 2nitrosopyridine calculated at DSD-PBEP86D3(BJ)/def2TZVP level of theory are very similar. Although there are some differences in frequencies and intensities of main bands, they are not significant enough to be useful for the analysis of the experimental spectra. Anharmonic spectra calculated at the same level of theory are even closer in frequencies, but relative intensities of two conformers are quite different. The fact that the intensities of anti conformer closely resembles intensities of experimental spectrum, and those of syn conformer do not, suggests dominant presence of anti conformer. Additional evidence in favor of the prevailing formation of anti conformer comes from the anharmonic spectra of 4-chloro-2-nitrosopyridine. At DSD-PBEP86-D3(BJ)/def2-TZVP level of theory third strongest band of anti conformer's spectrum is located at $705 \mathrm{~cm}^{-1}$, close to the experimental frequency of $701 \mathrm{~cm}^{-1}$, while in syn conformer's spectrum the same band is shifted to $764 \mathrm{~cm}^{-1}$. In experimental spectrum there are no bands around $764 \mathrm{~cm}^{-1}$.

To estimate a degree of similarity between calculated and experimental frequencies, and to minimize a possibility of incorrect assignations, several strongest and 
relatively isolated bands in experimental IR spectra of three anti-2-nitrosopyridines (1-3) and previously recorded spectrum of nitrosobenzene ${ }^{[3]}$ were selected for comparison with the calculated frequencies. These eight reference bands are the strong band close to $1500 \mathrm{~cm}^{-1}$ in each of four experimental spectra, band at around $1150 \mathrm{~cm}^{-1}$ in all spectra except in that of 4-methyl-2-nitrosopyridine, and relatively strong and isolated band at $701 \mathrm{~cm}^{-1}$ in the spectrum of 4-chloro-2-nitrosopyridine.

Decision which band in scaled harmonic spectra corresponds to particular reference band was not in all cases straightforward, or even possible. Spectra obtained with several of the density functionals in the $\mathrm{N}=\mathrm{O}$ stretching region are so different from both the experimental spectra and spectra obtained with other functionals, that any choice would be arbitrary. To help the objective assignations, compositions of normal modes whose scaled frequencies were reasonably close to the frequencies of reference bands were analyzed using the program vibAnalysis, ${ }^{[8,9]}$ and the results compared with the results for other functionals. All of the decisions were thus based upon estimates of normal modes frequencies, compositions and relative intensities.

In calculated spectra of all nitroso compounds, region between 1450 and $1650 \mathrm{~cm}^{-1}$ contains several bands, one or two of which are among the strongest bands in spectrum. Vibrational analysis reveals that they belong to normal modes with at least some $\mathrm{N}=\mathrm{O}$ bond stretching contribution. In most cases, one mode has significantly higher $\mathrm{N}=\mathrm{O}$ stretching contribution than the others (40$70 \%)$, and often the highest intensity as well. In such cases, these bands were confidently selected for frequency comparison with the frequency of corresponding reference bands. However, in several instances calculated spectrum contains two or even three bands with similar intensities and similar $\mathrm{N}=\mathrm{O}$ stretching contributions. Although such patterns are in disagreement with the results of other functionals, just for the orientation, their average frequency was nonetheless compared with the reference frequency.

All calculated spectra contain an isolated band in second spectral region, between 1100 and $1150 \mathrm{~cm}^{-1}$, which by both frequency and intensity resembles experimental $1150 \mathrm{~cm}^{-1}$ band. Vibrational analysis of the corresponding normal mode shows that it is in-plane ring distortion with an addition of $8-14 \%$ of $\mathrm{C}-\mathrm{N}(=\mathrm{O})$ bond stretching. In case of 4-chloro-2-nitrosopyridine, it also contains $6-8 \%$ of $\mathrm{C}-\mathrm{Cl}$ bond stretching. Although it is present in the spectra of 4-methyl-2nitrosopyridine as well, the presence of neighboring bands of similar intensity make assignations uncertain.

And finally, in all calculated spectra of 4-chloro-2nitrosopyridine there is an easy identifiable, isolated band at around $700 \mathrm{~cm}^{-1}$, clearly resembling $701 \mathrm{~cm}^{-1}$ band in the experimental spectrum. Vibrational analysis shows that it belongs to in-plane ring deformation normal mode that contains $16-17 \%$ of $\mathrm{C}-\mathrm{Cl}$ bond stretching.

In Table 2 for each level of theory are given differences between calculated and experimental frequencies for the reference bands, corresponding RMSDs (root mean square deviations), and scaling factors used for conversion of harmonic to fundamental frequencies. Scaling factors are either taken from the literature, ${ }^{[12,22]}$ or are new, determined in this paper. Additional scaling factors, not shown in Table 2, can be found in Supporting information, Table S1.

Actually, harmonic frequencies of all four compounds were calculated with larger number of functionals than shown in Table 2. For instance, in addition to TPSShD3(BJ) and DSD-PBEP86-D3(BJ), frequencies were also calculated with revTPSSh-D3(BJ) and revDSDPBEP86-D3(BJ). As both revised versions produce marginally larger RMSD values, only the original versions of these functionals are included in Table 2. In almost all examined cases, with the exception of PBEO-D3(BJ), dispersion-corrected ${ }^{[23]}$ forms of functionals have slightly lower RMSD values than their uncorrected forms, so the results for B3LYP, TPSSh, B2PLYP, etc., are also not shown in Table 2.

Based on differences between calculated frequencies and frequencies of selected experimental bands, measured by the corresponding RMSDs, density functionals shown in Table 2 can be divided into two groups. The first contains so-called double-hybrid (DH) functionals, belonging to fifth rung of Jacob's ladder, ${ }^{[24]}$ generally considered as the most accurate and universal methods for calculation of molecular properties. ${ }^{[25]}$ Scaled harmonic frequencies calculated with them, with the exception of PBEO-DH and $\mathrm{PBE}-\mathrm{QIDH}$, agree exceptionally well with the selected experimental frequencies, with average RMSD of just $15 \mathrm{~cm}^{-1}$. The smallest RMSD values have DSD-PBEP86-D3(BJ) $\left(11 \mathrm{~cm}^{-1}\right)$, B2GP-PLYP-D3(BJ) $\left(12 \mathrm{~cm}^{-1}\right)$, and mPW2-PLYP-D2 $\left(13 \mathrm{~cm}^{-1}\right)$. These excellent results support two conclusions. First, that DH functionals with def2-TZVP basis set are capable of producing accurate experimental frequencies of nitroso compounds, and second, that photolysis of 2-nitrosopyridine dimers indeed produces corresponding monomers.

The second group of functionals, comprising non-DH functionals, have four times higher average RMSD of $65 \mathrm{~cm}^{-1}$. The best of them (TPSSh-D3(BJ), B97-D3(BJ) and $B 3 L Y P-D 3(B J))$ have RMSDs between 32 and $39 \mathrm{~cm}^{-1}$, and the rest have average RMSD of $77 \mathrm{~cm}^{-1}$. What is the origin of these bad numbers? While average RMSD for $1150 \mathrm{~cm}^{-1}$ and $700 \mathrm{~cm}^{-1}$ bands for nonDH functionals is probably acceptable $\left(26 \mathrm{~cm}^{-1}\right)$, average RMSD for $1500 \mathrm{~cm}^{-1}$ bands is $85 \mathrm{~cm}^{-1}$, or $107 \mathrm{~cm}^{-1}$ without three outliers. As B3LYP$\mathrm{D} 3(\mathrm{BJ})$ and $\mathrm{B} 97-\mathrm{D} 3(\mathrm{BJ})$ have problems with reproducing the 
Table 2. Differences between calculated and experimental reference bands frequencies, corresponding RMSDs, and scaling factors $\left(\lambda_{F}\right)$ used for conversion of calculated harmonic to experimental fundamental frequencies.

\begin{tabular}{|c|c|c|c|c|c|c|c|c|c|c|c|}
\hline \multirow{3}{*}{ Method ${ }^{(a)}$} & \multirow{3}{*}{$\lambda_{\mathrm{F}}$} & \multirow{3}{*}{ lit.(b) } & \multicolumn{9}{|c|}{ Compounds and frequencies of selected reference bands (in $\mathrm{cm}^{--}$) } \\
\hline & & & \multicolumn{2}{|c|}{$1(\mathrm{R}=\mathrm{H})$} & \multicolumn{3}{|c|}{$2(\mathrm{R}=\mathrm{Cl})$} & \multirow{2}{*}{$\frac{3(\mathrm{R}=\mathrm{Me})}{1512}$} & \multicolumn{2}{|c|}{ Nitrosobenzene } & \multirow{2}{*}{ RMSD } \\
\hline & & & 1509 & 1150 & 1507 & 1160 & 701 & & 1503 & 1118 & \\
\hline \multicolumn{12}{|c|}{ non-DH functionals, scaled harmonic frequencies } \\
\hline B3LYP-D3(BJ) & c & T & $43^{(c)}$ & -34 & 66 & -38 & -22 & $39^{(c)}$ & 15 & -32 & 39 \\
\hline B97-D3(BJ) & 0.9867 & & 2 & $-65^{(c)}$ & 47 & -48 & -19 & -2 & 0 & -46 & 37 \\
\hline M06-2X & 0.946 & T & 137 & -21 & 142 & -24 & -22 & 92 & 130 & -32 & 91 \\
\hline MN15 & 0.9515 & & 118 & -14 & 122 & -20 & -31 & 115 & 52 & -32 & 77 \\
\hline PBEO & 0.9575 & & 114 & -12 & 116 & -20 & -16 & 111 & 52 & -23 & 73 \\
\hline PW6B95-D3(BJ) & 0.9542 & & 103 & -15 & 105 & -23 & -21 & $85^{(c)}$ & 46 & -26 & 64 \\
\hline TPSSh-D3(BJ) & 0.9686 & & 16 & -32 & 60 & -36 & -24 & 12 & 13 & -32 & 32 \\
\hline$\omega B 97 X-D$ & 0.950 & T & 112 & -18 & 116 & -21 & -18 & 110 & 54 & -24 & 73 \\
\hline$\omega B 97 X-V$ & 0.9539 & & 130 & -9 & 115 & -26 & -18 & 128 & 73 & -18 & 82 \\
\hline$\omega B 97 M-V$ & 0.9578 & & 123 & -11 & 114 & -20 & -17 & 123 & 70 & -21 & 79 \\
\hline \multicolumn{12}{|c|}{ non-DH functional, anharmonic frequencies } \\
\hline TPSSh-D3(BJ) & & & 19 & -23 & 18 & -23 & -10 & 29 & 28 & -27 & 23 \\
\hline \multicolumn{12}{|c|}{ MP2/SCS-MP2, scaled harmonic frequencies } \\
\hline MP2 & 0.9575 & M & -68 & -5 & -74 & -19 & -23 & -72 & -104 & -15 & 58 \\
\hline SCS-MP2 & 0.9589 & M & -45 & -6 & -48 & -14 & -22 & -49 & -29 & -14 & 33 \\
\hline \multicolumn{12}{|c|}{ DH functionals, scaled harmonic frequencies } \\
\hline B2GP-PLYL-D3(BJ) & 0.9543 & & -5 & -8 & -6 & -17 & -17 & -10 & -9 & -18 & 12 \\
\hline B2-PLYL-D3(BJ) & 0.9623 & M & -16 & $-19^{(c)}$ & -16 & -24 & -16 & -21 & $-48^{(c)}$ & -23 & 25 \\
\hline DSD-PBEP86-D3(BJ) & 0.9617 & M & -6 & -7 & -5 & -13 & -16 & -10 & -8 & -17 & 11 \\
\hline mPW2-PLYP-D2 & 0.9572 & & 4 & -13 & 4 & -21 & -17 & 1 & -1 & -20 & 13 \\
\hline revDSD-BLYP-D3(BJ) & 0.9565 & & -14 & -16 & -12 & -13 & -16 & -17 & -12 & -16 & 15 \\
\hline PBEO-DH & 0.9437 & & 109 & -2 & 111 & -9 & -16 & $92^{(c)}$ & 53 & -18 & 67 \\
\hline PBE-QIDH & 0.9421 & & 37 & 0 & 80 & -8 & -17 & $6^{(c)}$ & 32 & -16 & 41 \\
\hline \multicolumn{12}{|c|}{ DH functional, anharmonic frequencies } \\
\hline DSD-PBEP86-D3(BJ) & & & 17 & 10 & 18 & 4 & 4 & 18 & 25 & 1 & 15 \\
\hline
\end{tabular}

correct shape of the spectra, and TPSSh-D3(BJ) predicts too high $\mathrm{N}=\mathrm{O}$ stretching frequency of 4-chloro-2-nitrosopyridine, it can be concluded that none of non-DH density functionals shown in Table 2 are capable of producing acceptable frequencies of all investigated nitrosoaromatic compounds. To see whether this is just the feature of nitrosoaromatic compounds, we have calculated vibrational frequencies of nonaromatic nitroso compound, $\mathrm{CH}_{3} \mathrm{NO}$ (Table $\mathrm{S} 2$ in Supporting information), for which vibrational spectrum is already known. ${ }^{[26]}$ Average of
RMSDs for $\mathrm{N}=\mathrm{O}$ stretching band obtained with B3LYPD3(BJ), B97-D3(BJ) and TPSSh-D3(BJ) functionals is $40 \mathrm{~cm}^{-1}$, with other non-DH functionals it is $97 \mathrm{~cm}^{-1}$, and with $\mathrm{DH}$ functionals (without two outliers) it is just $9 \mathrm{~cm}^{-1}$. It can be safely concluded that the origin of the problem is in the $\mathrm{N}=\mathrm{O}$ bond itself, and has nothing to do with the rest of the molecule. The inability of non-DH (and some $\mathrm{DH}$ ) density functionals to reproduce the $\mathrm{N}=\mathrm{O}$ stretching frequency can be ascribed to their inadequate quantum-mechanical description of $\mathrm{N}=\mathrm{O}$ group, which is probably the 
consequence of its known multireference character. ${ }^{[27]}$

We have also tested MP2 and SCS-MP2 methods. The RMSD value for MP2 $\left(58 \mathrm{~cm}^{-1}\right)$ is somewhat lower than average non-DH DFT RMSD, but SCS-MP2 $\left(33 \mathrm{~cm}^{-1}\right)$ offers definitive improvement. However, since the result of SCSMP2 is only in the best non-DH DFT region, there is no real reason to use it.

To see whether there are any significant changes relative to the simpler and faster approach, we have calculated anharmonic spectra of all compounds with the best functional from both groups. From the resulting RMSD values reported in Table 2 it is clear that TPSSh-D3(BJ) anharmonic frequencies represent an improvement relative to the harmonic frequencies, but still not in the range of frequencies obtained with $\mathrm{DH}$ functionals. The anharmonic frequencies obtained with DSD-PBEP86-D3(BJ) functional produce practically the same RMSD value $\left(15 \mathrm{~cm}^{-1}\right)$ as do the scaled harmonic ones $\left(11 \mathrm{~cm}^{-1}\right)$, leading to the conclusion that tedious calculation of anharmonic frequencies with $\mathrm{DH}$ functionals, at least for the compounds investigated in this paper, does not necessarily offer the advantage relative to the simpler approach of multiplying calculated harmonic frequencies with the single scaling factor specific for the level of theory.

There is a feature common to all scaled harmonic frequencies calculated with $\mathrm{DH}$ functionals, not counting two flawed frequencies obtained with PBEO-DH and PBEQIDH. Corresponding differences shown in Table 2 are all negative, with average value of $-10 \mathrm{~cm}^{-1}$, meaning that all frequencies are slightly underestimated by one-parameter $\left(I_{F}\right)$ uniform scaling procedure [Eq. (1)]. This suggests that possibly better results can be obtained with linear scaling equation approach ${ }^{[14]}$ [Eq. (2)].

$$
\begin{aligned}
& \tilde{v}_{\text {exp }}=\lambda_{\mathrm{F}} \tilde{v}_{\text {calc }} \\
& \tilde{v}_{\text {exp }}=a \tilde{v}_{\text {calc }}+b
\end{aligned}
$$

Parameters $a$ and $b$ for DSD-PBEP86-D3(BJ)/def2TZVP level of theory were determined by linear correlation of calculated harmonic and experimental fundamental frequencies of Martin's dataset, ${ }^{[12]}$ and then used for scaling harmonic frequencies of four molecules examined in this paper. As the new frequency differences are smaller and of both signs, their RMSD value is reduced to $3 \mathrm{~cm}^{-1}$, and their average value of $-2 \mathrm{~cm}^{-1}$ is much closer to zero.

In Figure 3 are compared differential spectra recorded after photolysis of dimers with the spectra of monomers 1-3 calculated using DSD-PBEP86-D3(BJ)/def2-TZVP harmonic frequencies scaled by just outlined procedure.

There is an excellent agreement between experimental and scaled harmonic spectra, with almost exact match between most of the corresponding bands. Not only in frequencies, but in relative intensities as well. Small number of bands present in the calculated spectra are either missing in the experimental spectra or have smaller intensities than predicted, which in principle might have to do with the interactions in the solid phase. Most notably, these are the bands close to $1600 \mathrm{~cm}^{-1}$, just above strong $\mathrm{N}=\mathrm{O}$ stretching bands, which belong to skeletal in-plane deformations of aromatic ring, usually with small addition of $\mathrm{N}=\mathrm{O}$ stretching vibration. Conversely, just two not so strong bands in three experimental spectra do not have apparent counterparts neither in scaled harmonic nor anharmonic spectra $-1131 \mathrm{~cm}^{-1}$ band in the spectrum of 1 and $977 \mathrm{~cm}^{-1}$ band in the spectrum of 3 . In other words, scaled harmonic spectra calculated with DSD-PBEP86D3(BJ) DH functional and def2-TZVP basis set can evidently serve as a good starting point for more detailed assignation of experimental spectra of nitrosoaromatic compounds.

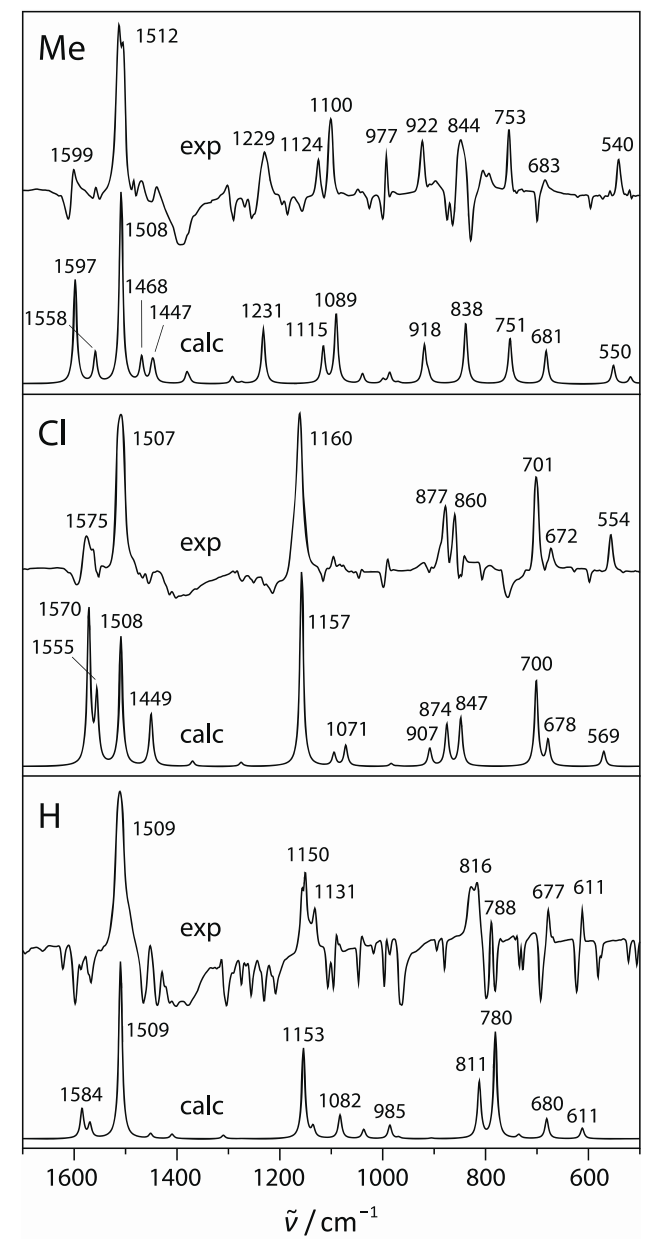

Figure 3. Comparison of the experimental differential FTIR spectra with the theoretical IR spectra of monomers 1-3, calculated using DSD-PBEP86-D3(BJ)/def2-TZVP harmonic frequencies scaled by two-parameter linear scaling [Eq. (2)]. 


\section{CONCLUSIONS}

Excellent agreement between experimental and theoretical IR spectra calculated with DH functionals and def2-TZVP basis set, confirms that photolysis of 2-nitrosopyridine dimers at $12 \mathrm{~K}$ produces corresponding monomers. The best results were obtained with DSD-PBEP86-D3(BJ), B2GP-PLYP$\mathrm{D} 3(\mathrm{BJ})$ and $\mathrm{mPW}$ 2-PLYP-D2 functionals.

Surprisingly, non-DH functionals, including otherwise very good $\omega \mathrm{B} 97 \mathrm{X}-\mathrm{V}^{[28]}$ and $\omega \mathrm{B} 97 \mathrm{M}-\mathrm{V}^{[29]}$ functionals, have serious problems with normal modes containing $\mathrm{N}=\mathrm{O}$ bond stretching vibration. As the same holds for nonaromatic nitrosomethane, the origin of the problem is clearly in the nitroso group itself, most likely in its inadequate quantum-mechanical description due to its multireference character.

For the compounds investigated in this paper, anharmonic corrections do not necessarily end up in frequencies closer to the experiment. The best scaled harmonic frequencies of 2-nitrosopyridines (1-3) and nitrosobenzene among tested non-DH functionals belong to TPSSh-D3(BJ), B97-D3(BJ) and B3LYP-D3(BJ) functionals and def2-TZVP basis set. While anharmonic corrections improve TPSSh-D3(BJ) frequencies, the effect on frequencies obtained with two other functionals is the opposite one. Similarly, scaled harmonic frequency of nitrosomethane $\mathrm{N}=\mathrm{O}$ stretching vibration is closer to the experimental value than its anharmonic equivalent. In the case of the best DH functional, DSD-PBEP86-D3(BJ), and several other $\mathrm{DH}$ functionals as well, there are no significant differences between scaled harmonic and anharmonic frequencies and intensities.

Acknowledgment. This work has been fully supported by Croatian Science Foundation under the project IP-2020-024467.

Supplementary Information. Supporting information to the paper is attached to the electronic version of the article at: https://doi.org/10.5562/cca3820.

PDF files with attached documents are best viewed with Adobe Acrobat Reader which is free and can be downloaded from Adobe's web site.

\section{REFERENCES}

[1] H. Vančik, Aromatic C-nitroso Compounds, Springer, Dordrecht, 2013.

[2] H. Vančik, V. Šimunić-Mežnarić, I. Čaleta, E. Meštrović, S. Milovac, J. Phys. Chem. B 2002, 106, 1576-1580. https://doi.org/10.1021/jp0115289

[3] A. Knežević, T. Medančić, S. Milovac, I. Biljan, I. Halasz, H. Vančik, Croat. Chem. Acta 2011, 84, 21-24. https://doi.org/10.5562/cca1714

[4] K. Varga, I. Biljan, V. Tomišić, Z. Mihalić, H. Vančik, J. Phys. Chem. A 2018, 122, 2542-2549. https://doi.org/10.1021/acs.jpca.7b12179
[5] Gaussian 16, Revision B.01, M. J. Frisch, G. W. Trucks, H. B. Schlegel, G. E. Scuseria, M. A. Robb, J. R. Cheeseman, G. Scalmani, V. Barone, G. A. Petersson, H. Nakatsuji, X. Li, M. Caricato, A. V. Marenich, J. Bloino, B. G. Janesko, R. Gomperts, B. Mennucci, H. P. Hratchian, J. V. Ortiz, A. F. Izmaylov, J. L. Sonnenberg, D. Williams-Young, F. Ding, F. Lipparini, F. Egidi, J. Goings, B. Peng, A. Petrone, T. Henderson, D. Ranasinghe, V. G. Zakrzewski, J. Gao, N. Rega, G. Zheng, W. Liang, M. Hada, M. Ehara, K. Toyota, R. Fukuda, J. Hasegawa, M. Ishida, T. Nakajima, Y. Honda, O. Kitao, H. Nakai, T. Vreven, K. Throssell, J. A. Montgomery, Jr., J. E. Peralta, F. Ogliaro, M. J. Bearpark, J. J. Heyd, E. N. Brothers, K. N. Kudin, V. N. Staroverov, T. A. Keith, R. Kobayashi, J. Normand, K. Raghavachari, A. P. Rendell, J. C. Burant, S. S. Iyengar, J. Tomasi, M. Cossi, J. M. Millam, M. Klene, C. Adamo, R. Cammi, J. W. Ochterski, R. L. Martin, K. Morokuma, O. Farkas, J. B. Foresman, D. J. Fox, Gaussian, Inc., Wallingford CT, 2016.

[6] F. Neese, F. Wennmohs, U. Becker, C. Riplinger, J. Chem. Phys. 2020, 152, 224108.

https://doi.org/10.1063/5.0004608

[7] Y. Shao, Z. Gan, E. Epifanovsky, A. T. B. Gilbert, M. Wormit, J. Kussmann, A. W. Lange, A. Behn, J. Deng, X. Feng, D. Ghosh, M. Goldey P. R. Horn, L. D. Jacobson, I. Kaliman, R. Z. Khaliullin, T. Kús, A. Landau, J. Liu, E. I. Proynov, Y. M. Rhee, R. M. Richard, M. A. Rohrdanz, R. P. Steele, E. J. Sundstrom, H. L. Woodcock III, P. M. Zimmerman, D. Zuev, B. Albrecht, E. Alguire, B. Austin, G. J. O. Beran, Y. A. Bernard, E. Berquist, K. Brandhorst, K. B. Bravaya, S. T. Brown, D. Casanova, C.-M. Chang, Y. Chen, S. H. Chien, K. D. Closser, D. L. Crittenden, M. Diedenhofen, R. A. DiStasio Jr., H. Dop, A. D. Dutoi, R. G. Edgar, S. Fatehi, L. FustiMolnar, A. Ghysels, A. Golubeva-Zadorozhnaya, J. Gomes, M. W. D. Hanson-Heine, P. H. P. Harbach, A. W. Hauser, E. G. Hohenstein, Z. C. Holden, T.-C. Jagau, H. Ji, B. Kaduk, K. Khistyaev, J. Kim, J. Kim, R. A. King, P. Klunzinger, D. Kosenkov, T. Kowalczyk, C. M. Krauter, K. U. Lao, A. Laurent, K. V. Lawler, S. V. Levchenko, C. Y. Lin, F. Liu, E. Livshits, R. C. Lochan, A. Luenser, P. Manohar, S. F. Manzer, S.-P. Mao, N. Mardirossian, A. V. Marenich, S. A. Maurer, N. J. Mayhall, C. M. Oana, R. Olivares-Amaya, D. P. O'Neill, J. A. Parkhill, T. M. Perrine, R. Peverati, P. A. Pieniazek, A. Prociuk, D. R. Rehn, E. Rosta, N. J. Russ, N. Sergueev, S. M. Sharada, S. Sharmaa, D. W. Small, A. Sodt, T. Stein, D. Stück, Y.-C. Su, A. J. W. Thom, T. Tsuchimochi, L. Vogt, O. Vydrov, T. Wang, M. A. Watson, J. Wenzel, A. White, C. F. Williams, V. Vanovschi, S. Yeganeh, S. R. Yost, Z.-Q. You, I. Y. Zhang, X. Zhang, Y. Zhou, B. R. Brooks, G. K. L. Chan, D. M. Chipman, C. J. Cramer, W. A. Goddard III, M. S. Gordon, W. J. Hehre, A. Klamt, H. F. Schaefer III, M. W. Schmidt, C. D. Sherrill, D. G. Truhlar, A. Warshel, X. Xua, A. Aspuru-Guzik, R. Baer, A. T. Bell, N. A. Besley, J.-D. Chai, A. Dreuw, B. D. Dunietz, T. R. Furlani, S. R. Gwaltney, C.-P. Hsu, Y. Jung, 
J. Kong, D. S. Lambrecht, W. Liang, C. Ochsenfeld, V. A. Rassolov, L. V. Slipchenko, J. E. Subotnik, T. Van Voorhis, J. M. Herbert, A. I. Krylov, P. M. W. Gill, M. Head-Gordon, Mol. Phys. 2015, 113, 184.

[8] F. Texiera, VibAnalysis-Tools for performing vibrational analysis on molecular systems, 2017; https://github.com/teixeirafilipe/vibAnalysis

[9] F. Teixeira, M. N. D. S. Cordeiro, J. Chem. Theory Comput. 2019, 15, 456-470.

https://doi.org/10.1021/acs.jctc.8b00439

[10] GaussView, Version 6.0, R. Dennington, T. A. Keith, J. M. Millam, Semichem Inc., Shawnee Mission, KS, 2016.

[11] DataGraph, Version 4.6; https://www.visualdatatools.com/DataGraph

[12] M. K. Kesharwani, B. Brauer, J. M. L. Martin, J. Phys. Chem. A 2015, 119, 1701-1714. https://doi.org/10.1021/jp508422u

[13] B. G. Gowenlock, M. J. Maidment, K. G. Orrell, V. Šik, G. M, G. Vasapollo, M. B. Hursthouse, K. M. A. Malik, J. Chem. Soc., Perkin Trans. 2 2000, 2280.

[14] M. A. Palafox, Phys. Sci. Rev. 2018, 3, 1. https://doi.org/10.1515/psr-2017-0184

[15] a) V. J. Barone, Chem. Phys. 2005, 122, 1. https://doi.org/10.1063/1.1824881

b) V. Barone, M. Biczysko, J. Bloino, Phys. Chem. Chem. Phys. 2014, 16, 1759-1787. https://doi.org/10.1039/c3cp53413h

[16] M. Swart, Results for the 2020 edition of the Annual DFT Popularity Poll: Primera Divisió; https://www.marcelswart.eu/dft-poll/res2020_q1.html

[17] L. Goerigk, A. Hansen. C. A. Bauer, S. Ehrlich, A. Najibi and S. Grimme, Phys. Chem. Chem. Phys. 2017, 19, 32184-32215.

https://doi.org/10.1039/C7CP04913G

[18] W. J. Hehre, L. Radom, P. v. R. Schleyer, J. A. Pople, Ab Initio Molecular Orbital Theory, New York 1986, John Wiley \& Sons.
[19] (a) T. H. Jr. Dunning, J. Chem. Phys. 1989, 90, 1007. https://doi.org/10.1063/1.456153

(b) A. K. Wilson, D. E. Woon, K. A. Peterson, T. H. Jr. Dunning, J. Chem. Phys. 1999, 110, 7667.

https://doi.org/10.1063/1.478678

[20] F. Jensen, J. Chem. Theory Comput. 2014, 10, 1074-1085. https://doi.org/10.1021/ct401026a

[21] F. Weigend, R. Ahlrichs, Phys. Chem. Chem. Phys. 2005, 7, 3297-3305. https://doi.org/10.1039/b508541a

[22] I. M. Alecu, J. Zheng, Y. Zhao, D. G. Truhlar, J. Chem. Theory Comput. 2010, 6, 2872-2887. https://doi.org/10.1021/ct100326h

[23] a) S. Grimme, S. Ehrlich, L. Goerigk, J. Comput. Chem. 2011, 32, 1456-1465. https://doi.org/10.1002/jcc.21759 b) S. Grimme, J. Antony, S. Ehrlich, H. Krieg, J. Chem. Phys. 2010, 132, 154104. https://doi.org/10.1063/1.3382344

[24] J. P. Perdew, K. Schmidt, AIP Conf. Proc. 2001, 577, 1. https://doi.org/10.1063/1.1390175

[25] L. Goerigk, S. Grimme, WIREs Comput. Mol. Sci. 2014, 4, 576-600. https://doi.org/10.1002/wcms.1193

[26] A. J. Barnes, H. E. Hallam, S. Waring, J. R. Armstrong, J. Chem. Soc, Faraday Trans. 21976 , 72, 1-10. https://doi.org/10.1039/F29767200001

[27] E. K. Dolgov, V. A. Bataev, I. A. Godunov, Int. J. Quant. Chem. 2004, 96, 193-201. https://doi.org/10.1002/qua.10683

[28] N. Mardirossian, M. Head-Gordon, Phys. Chem. Chem. Phys. 2014, 16, 9904-9924. https://doi.org/10.1039/c3cp54374a

[29] N. Mardirossian, M. Head-Gordon, J. Chem. Phys. 2016, 144, 214110. https://doi.org/10.1063/1.4952647 